Supporting Information

for

\title{
Controls of Microstructure and Chemical Reactivity on the Replacement of Limestone by Fluorite Studied Using Spatially Resolved Small Angle X-ray and Neutron Scattering
}

Juliane Weber ${ }^{1 *}$, Michael C. Cheshire ${ }^{1}$, Victoria H. Distefano ${ }^{1,2}$, Kenneth C. Littrell ${ }^{1}$, Jan Ilavsky $^{3}$, Markus Bleuel ${ }^{4}$, Jessica K. Bozell-Messerschmidt ${ }^{5}$, Anton Ievlev ${ }^{6}$, Andrew G. Stack ${ }^{1}$, Lawrence A. Anovitz ${ }^{1}$

${ }^{1}$ Chemical Science Division, Oak Ridge National Laboratory, Oak Ridge, USA.

${ }^{2}$ University of Tennessee, Knoxville, USA.

${ }^{3}$ Argonne National Laboratory, Chicago, USA.

${ }^{4}$ National Institute for Standard and Technology (NIST), Gaithersburg, USA.

${ }^{5}$ University of Nebraska, Omaha, USA.

${ }^{6}$ Center for Nanophase Material Science, Oak Ridge National Laboratory, Oak Ridge, USA.

*Corresponding author: julianeweber@email.arizona.edu,julianeweber3@gmx.de 


\section{Table of Contents}

2. Porosity Determination for Reference Materials............................................................. 2

3. Additional SEM Characterization of WD Reference Sample ................................................ 3

4. Pore Size Distribution for Reference Materials.............................................................................. 4

5. Photographs of Thin Sections Prepared from Reacted Rock Cores .................................... 4

6. Reaction Extent Determination Using Weight Analyses.......................................................... 5

7. Wide-angle X-ray Scattering Spectra ….............................................................................. 7

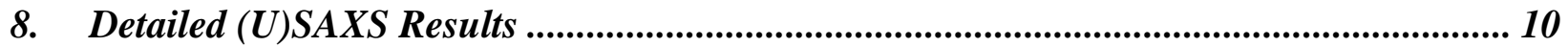

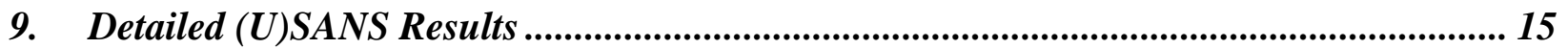

10. Pore Size Distribution for TC Limestone ……….......................................................... 18

11. Additional SEM Characterization ........................................................................... 19

12. Additional ToF-SIMS characterization ..................................................................... 21

13. Images Used in Rim Thickness Analyses....................................................................... 22

\section{Porosity Determination for Reference Materials}

Table S 1 Porosity Determination of Reference Materials via Weight Determination.

\begin{tabular}{|l|r|r|r|r|r|}
\hline Rock Name & Porosity $(\%)$ & Weight (dry) $(\mathrm{g})$ & $\begin{array}{l}\text { Weight } \\
(\text { wet })(\mathrm{g})\end{array}$ & $\begin{array}{l}\text { Diameter } \\
(\mathrm{cm})\end{array}$ & Height $(\mathrm{cm})$ \\
\hline T 1 & 23.63 & 5.67 & 6.36 & 1.56464 & 1.52908 \\
\hline T 2 & 22.4 & 6.09 & 6.81 & 1.59258 & 1.59512 \\
\hline T 3 & 23.6 & 5.99 & 6.73 & 1.58242 & 1.55956 \\
\hline T 4 & 22.4 & 5.1 & 5.73 & 1.56718 & 1.40208 \\
\hline T 5 & 21.97 & 6.28 & 7 & 1.59258 & 1.63322 \\
\hline T 6 & 21.63 & 6.3 & 7.04 & 1.60274 & 1.6637 \\
\hline T 7 & 22.86 & 6 & 6.72 & 1.55702 & 1.65354 \\
\hline T 8 & 21.62 & 6.18 & 6.9 & 1.57734 & 1.68402 \\
\hline CM 1 & 1.9 & 8.09 & 8.15 & 1.62306 & 1.5748 \\
\hline CM 2 & 2.2 & 7.98 & 8.05 & 1.62814 & 1.524 \\
\hline CM 3 & 1.7 & 8.13 & 8.18 & 1.62052 & 1.52908 \\
\hline CM 4 & 2 & 8.16 & 8.22 & 1.61544 & 1.52908 \\
\hline CM 5 & 1.8 & 8.62 & 8.69 & 1.62052 & 1.6129 \\
\hline CM 6 & 1.2 & 8.7 & 8.76 & 1.62052 & 1.62306 \\
\hline CM 7 & & 8.28 & 8.32 & 1.62052 & 1.5621 \\
\hline
\end{tabular}




\begin{tabular}{|l|r|r|r|r|r|} 
CM 8 & 1.8 & 9.02 & 9.08 & 1.63068 & 1.6891 \\
\hline W 1 & 4.7 & 8.74 & 8.9 & 1.62814 & 1.64846 \\
\hline W 2 & 2.8 & 8.31 & 8.4 & 1.63576 & 1.57734 \\
\hline W 3 & 2.8 & 8.21 & 8.3 & 1.6256 & 1.54178 \\
\hline W 4 & 3.1 & 8.18 & 8.28 & 1.62306 & 1.55448 \\
\hline
\end{tabular}

Texas cream (T), Carthage marble ( CM), Wisconsin dolomite (W)

\section{Additional SEM Characterization of WD Reference Sample}
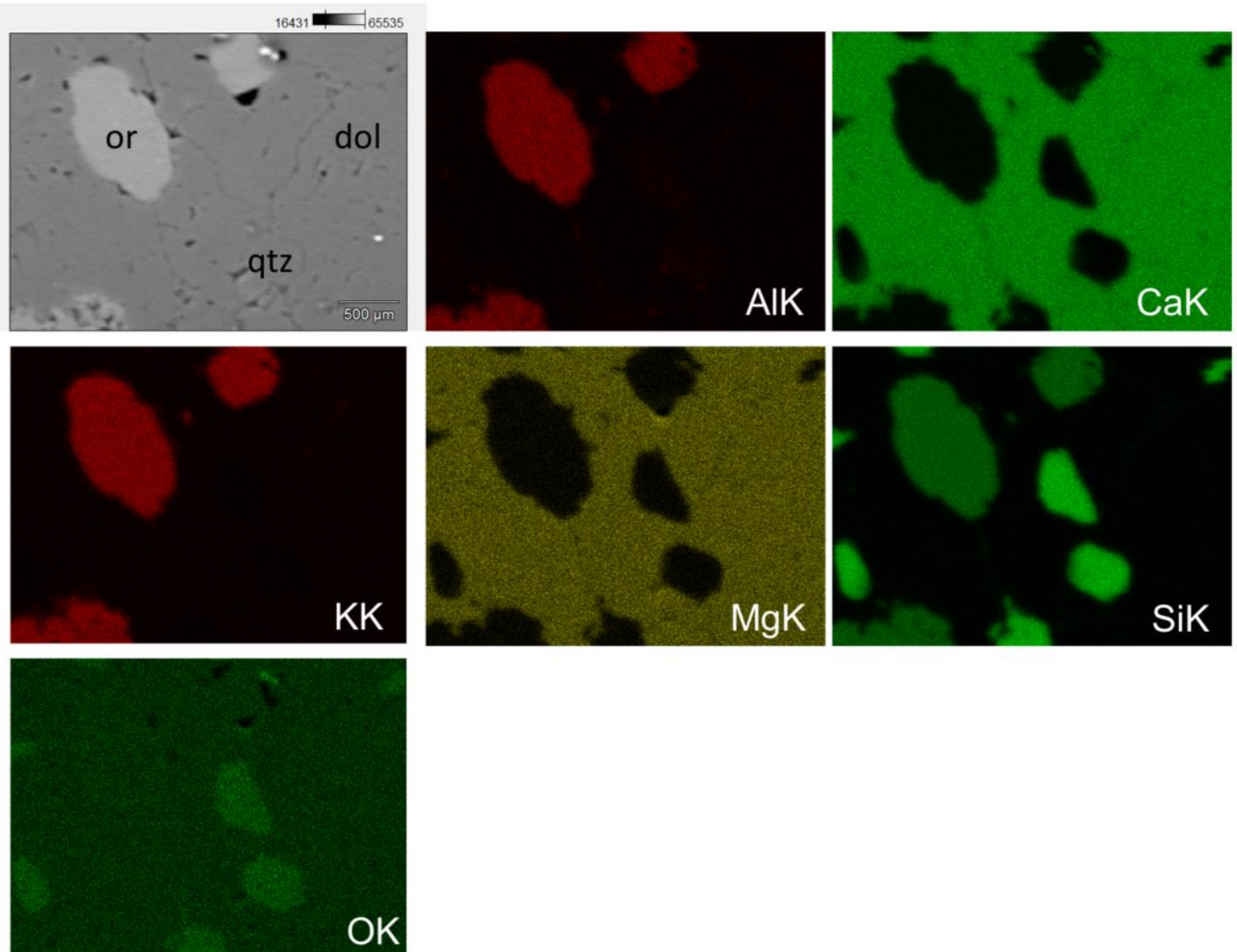

Figure S 1

SEM-EDS mapping of WD reference sample showing different gray scale features in BSE contrast image. By compositional mapping, these grains can be identified as orthoclase (or, chemical composition: $\left.\mathrm{KAISi}_{3} \mathrm{O}_{8}\right)$ and quartz (qtz, chemical composition: $\mathrm{SiO}_{2}$ ) grains in a dolomite (dol, chemical composition: $\left.\mathrm{CaMg}\left(\mathrm{CO}_{3}\right)_{2}\right)$ matrix. 


\section{Pore Size Distribution for Reference Materials}

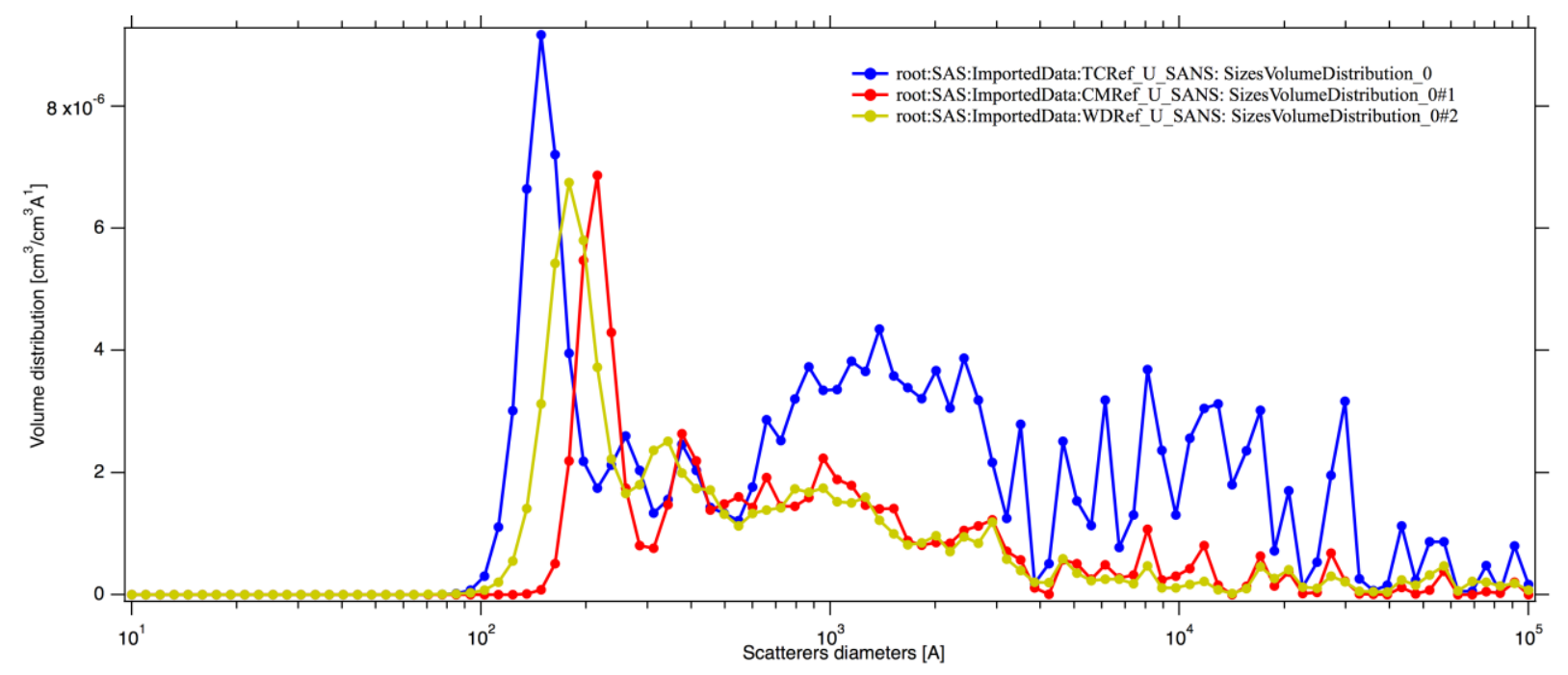

Figure S 2 Pore Size Distribution for Reference TC, CM and WD rocks prior to reaction with fluorite.

\section{Photographs of Thin Sections Prepared from Reacted Rock Cores}

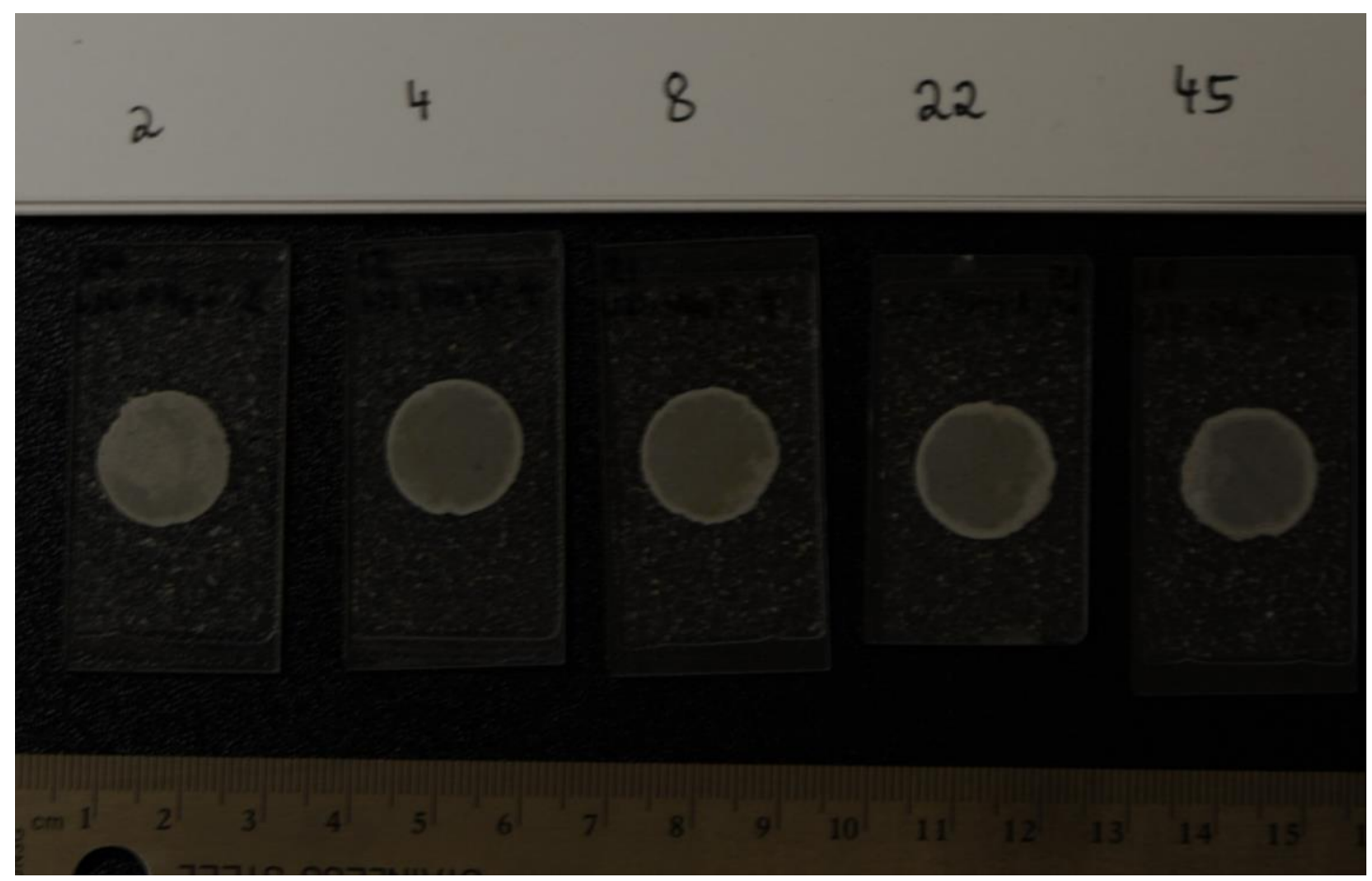




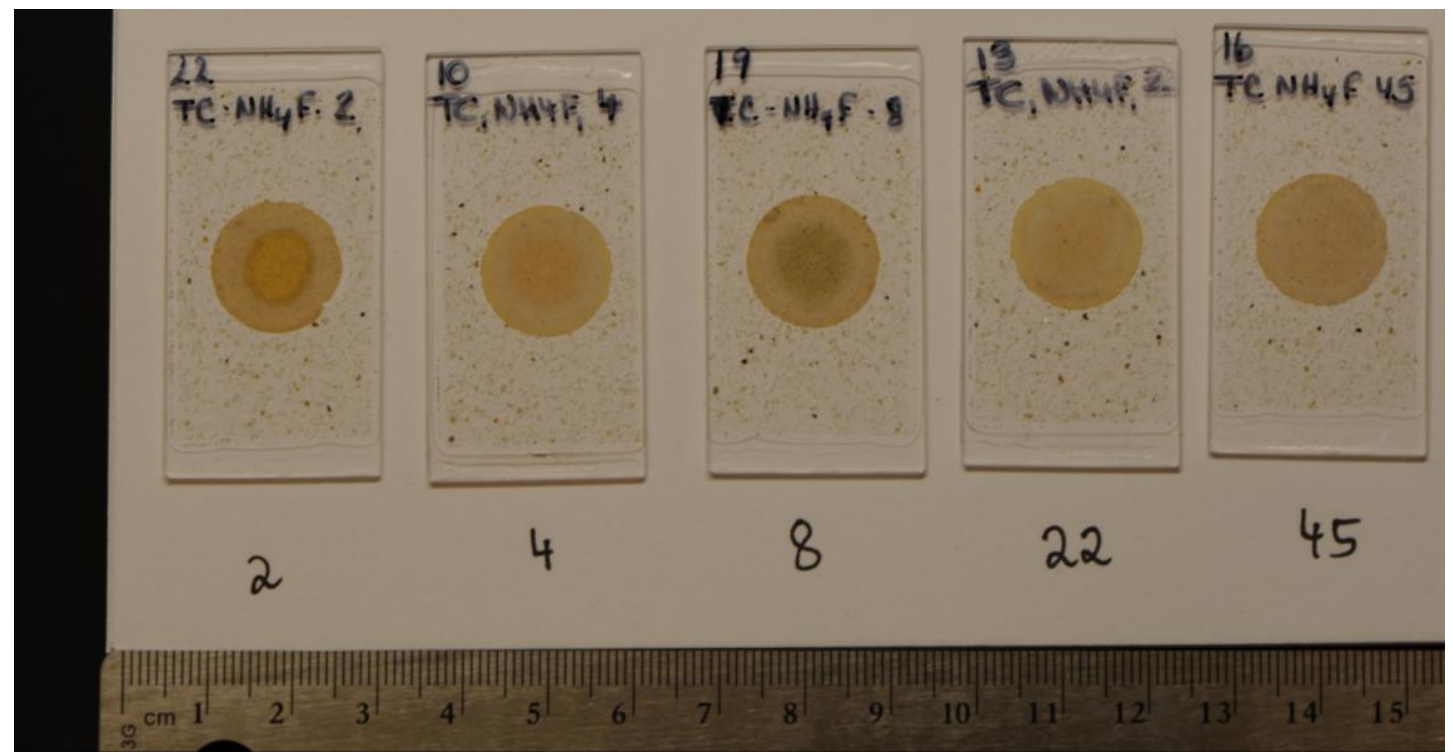

Figure S $4 \quad$ Thin sections prepared from TC limestone rock cores reacted with saturated $\mathrm{NH}_{4} \mathrm{~F}$ solution.

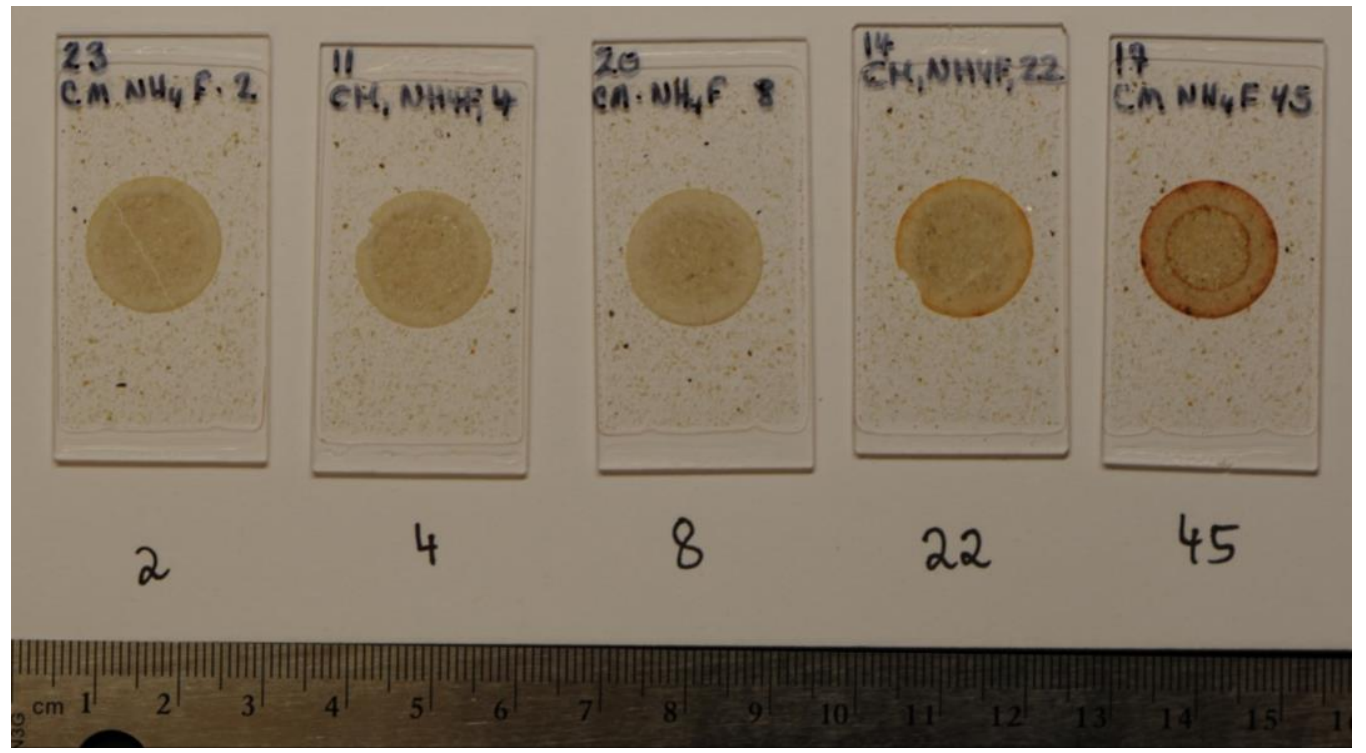

Figure S $5 \quad$ Thin sections prepared from $\mathrm{CM}$ limestone rock cores reacted with saturated $\mathrm{NH}_{4} \mathrm{~F}$ solution.

\section{Reaction Extent Determination Using Weight Analyses}

Table S $2 \quad$ Reaction extent calculation based on weight changes of solid samples.

$\begin{array}{lcccc}\begin{array}{c}\text { Experiment } \\ \text { name }\end{array} & \begin{array}{c}\text { Reaction } \\ \text { time }[\mathbf{d}]\end{array} & \mathbf{m}_{\text {final }}[\mathbf{g}] & \mathbf{m}_{\text {initial }}[\mathbf{g}] & \boldsymbol{\Delta} \mathbf{m}[\mathbf{w t} \% \mathbf{0} \\ \text { TC2 } & 2 & 5.5600 & 4.3938 & -26.5 \\ \text { TC4 } & 4 & 5.7515 & 4.4336 & -29.7 \\ \text { TC8 } & 8 & 6.1169 & 5.0090 & -22.1 \\ \text { TC22 } & 22 & 5.8344 & 4.7600 & -22.6\end{array}$




$\begin{array}{lcccc}\text { TC45 } & 45 & 5.6118 & 4.2858 & -30.9 \\ \text { CM2 } & 2 & 8.0843 & 7.1526 & -13.0 \\ \text { CM4 } & 4 & 9.0321 & 7.9496 & -13.6 \\ \text { CM8 } & 8 & 7.8337 & 6.5707 & -19.2 \\ \text { CM22 } & 22 & 8.4449 & 6.1216 & -38.0 \\ \text { CM45 } & 45 & 7.8715 & 6.7106 & -17.3 \\ \text { WD2 } & 2 & 8.2904 & 7.7587 & -6.9 \\ \text { WD4 } & 4 & 7.8742 & 7.4140 & -6.2 \\ \text { WD8 } & 8 & 8.0619 & 7.2043 & -11.9 \\ \text { WD22 } & 22 & 9.1159 & 8.2704 & -10.2 \\ \text { WD45 } & 45 & 8.1950 & 6.9533 & -17.9\end{array}$

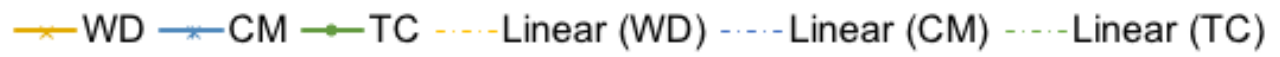

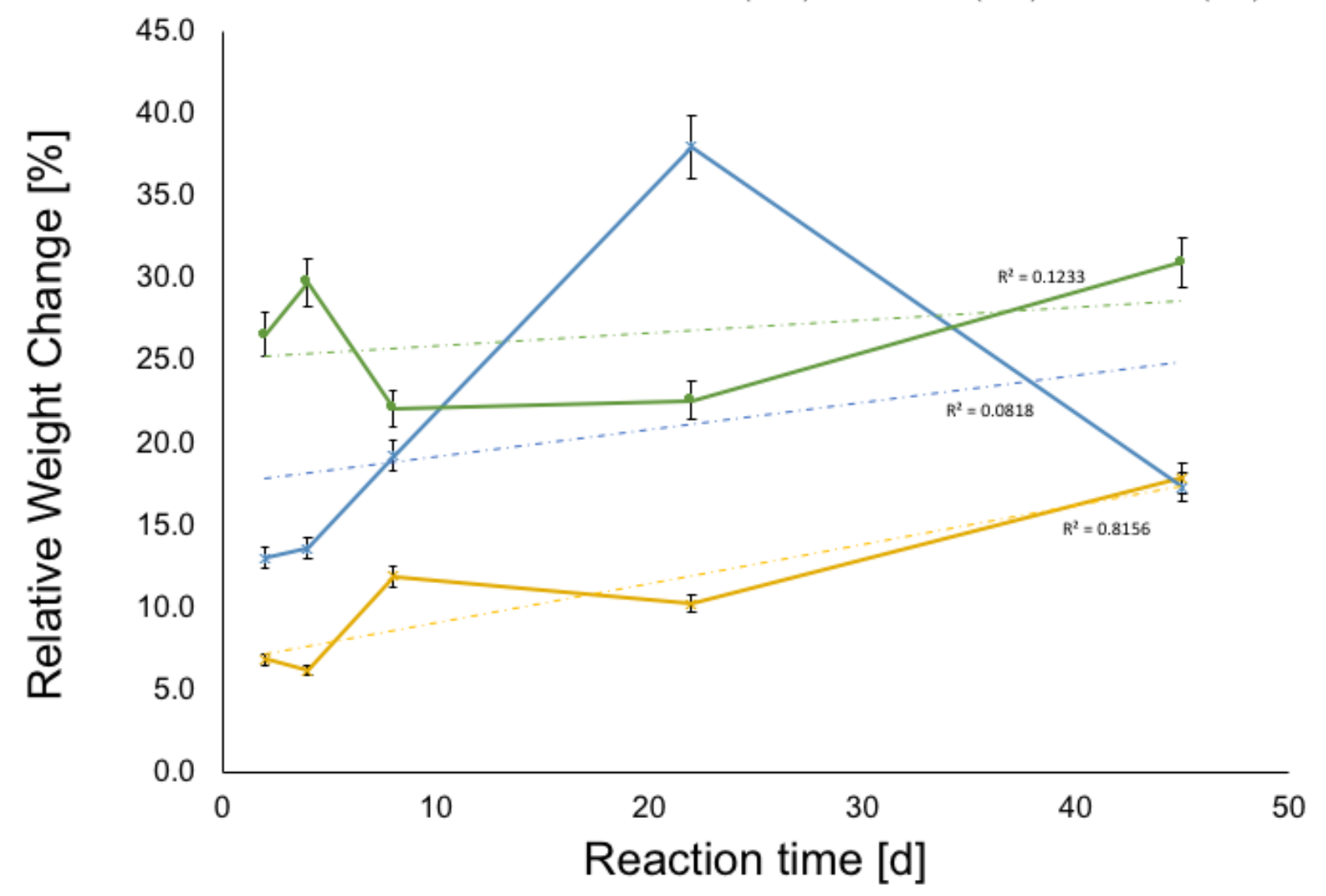

Figure S 6 Relative weight change of rock cores with reaction time for the three different rock types. 


\section{Wide-angle X-ray Scattering Spectra}

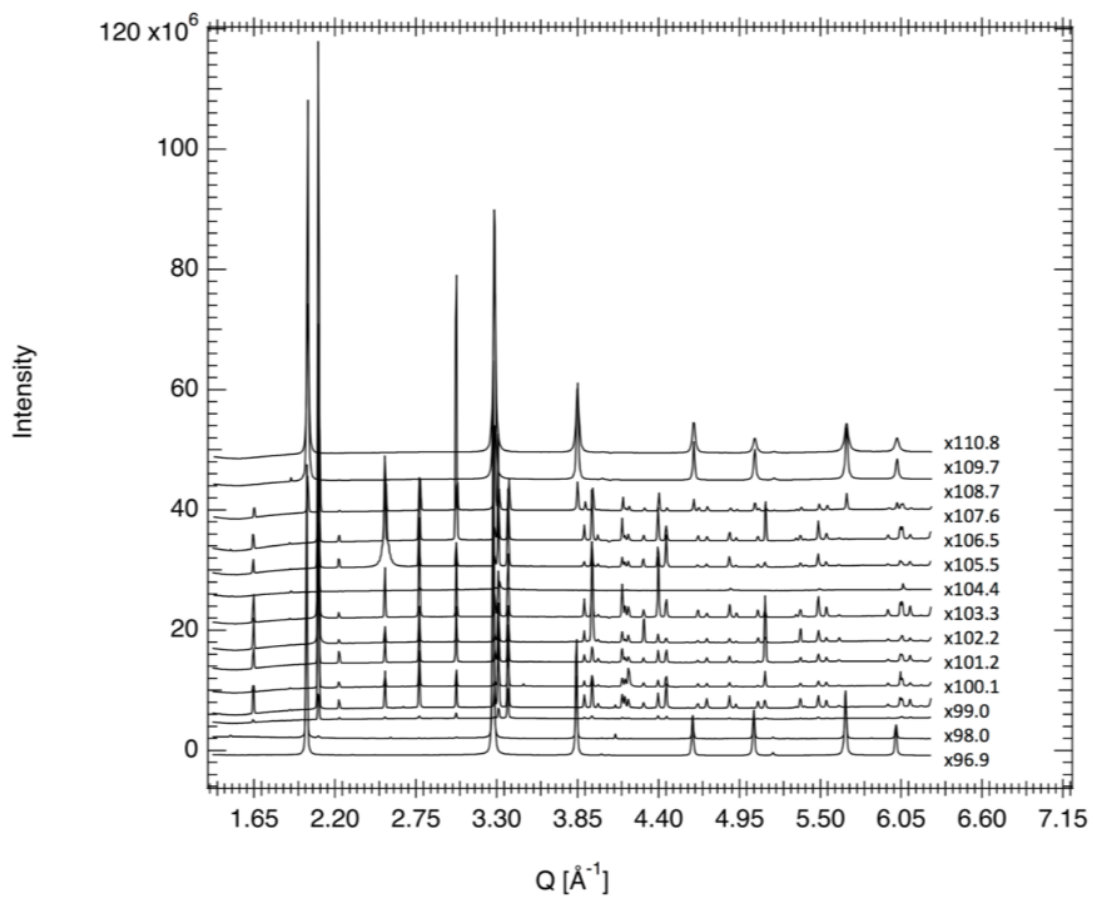

Figure S $7 \quad$ WAXS spectra for CMF4.

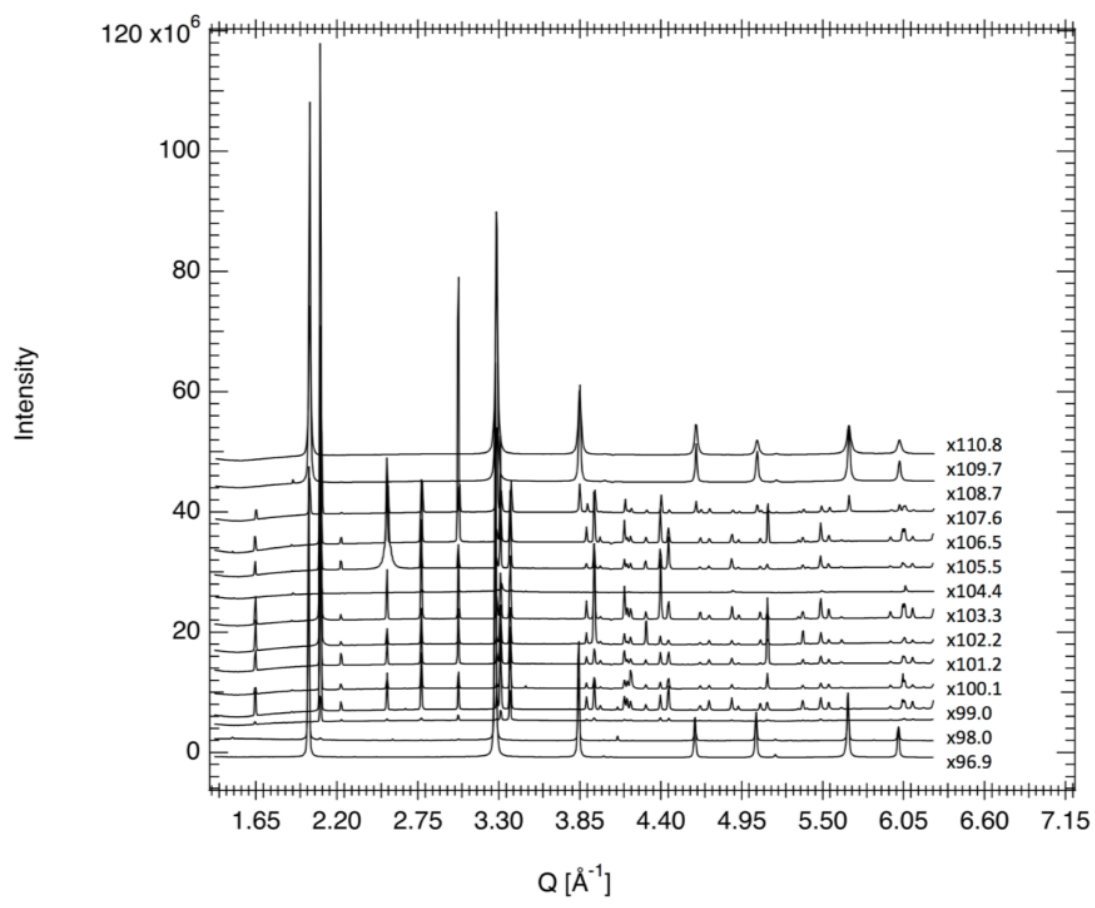

Figure S $8 \quad$ WAXS spectra for CMF8. 


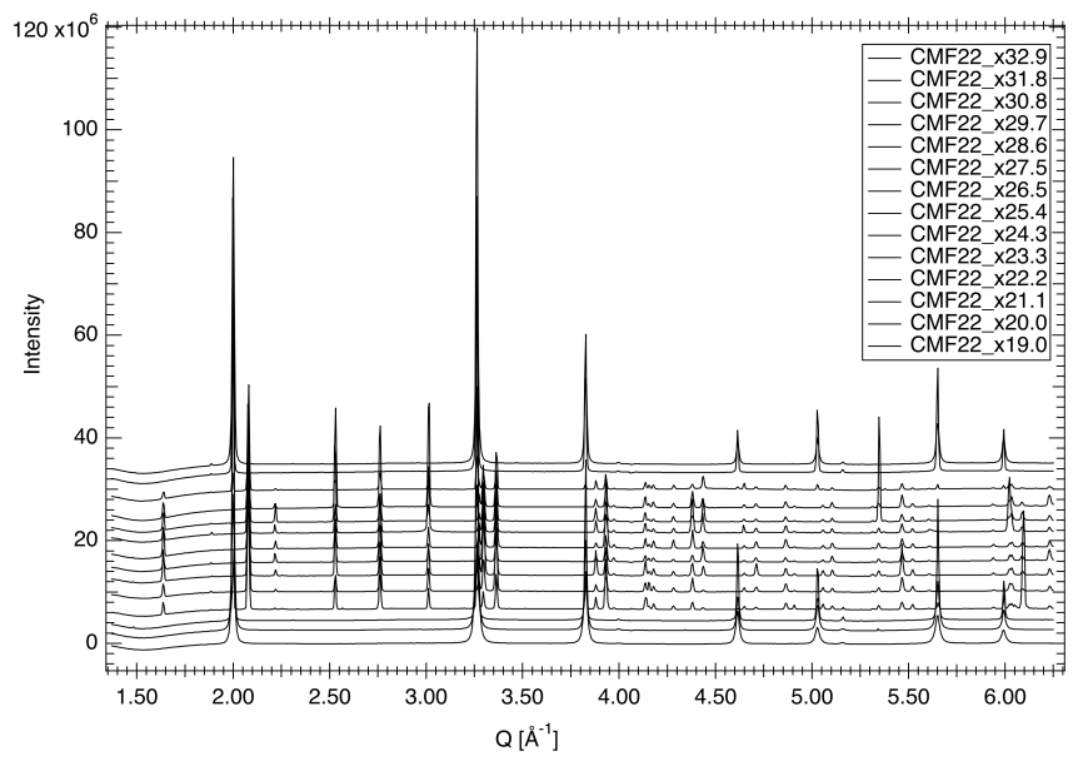

Figure S $9 \quad$ WAXS spectra for CMF22.

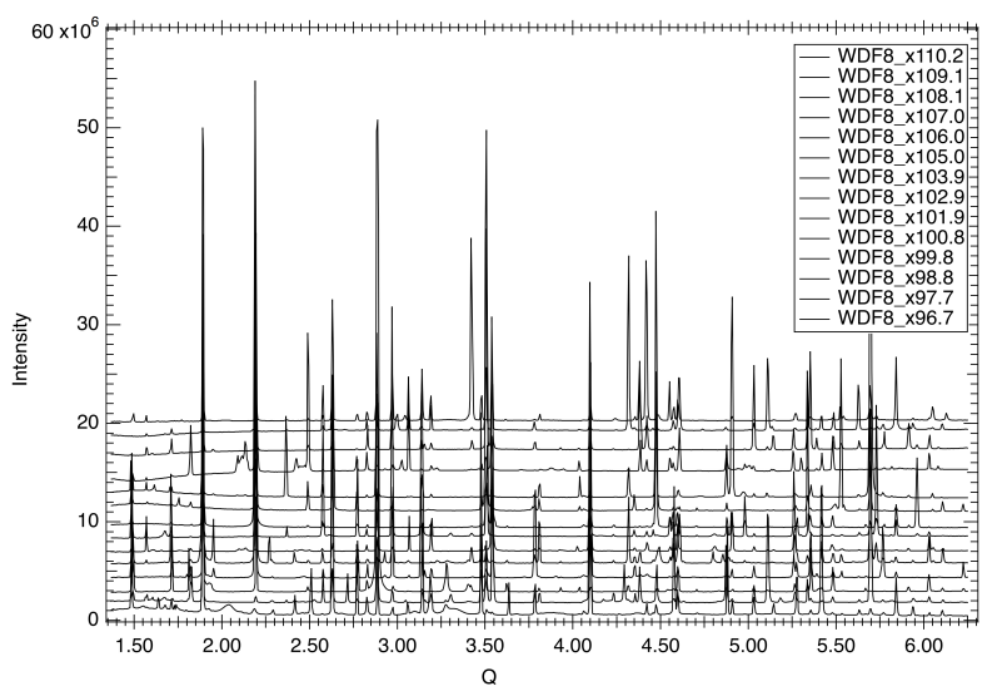

Figure S $10 \quad$ WAXS spectra for WDF8. 


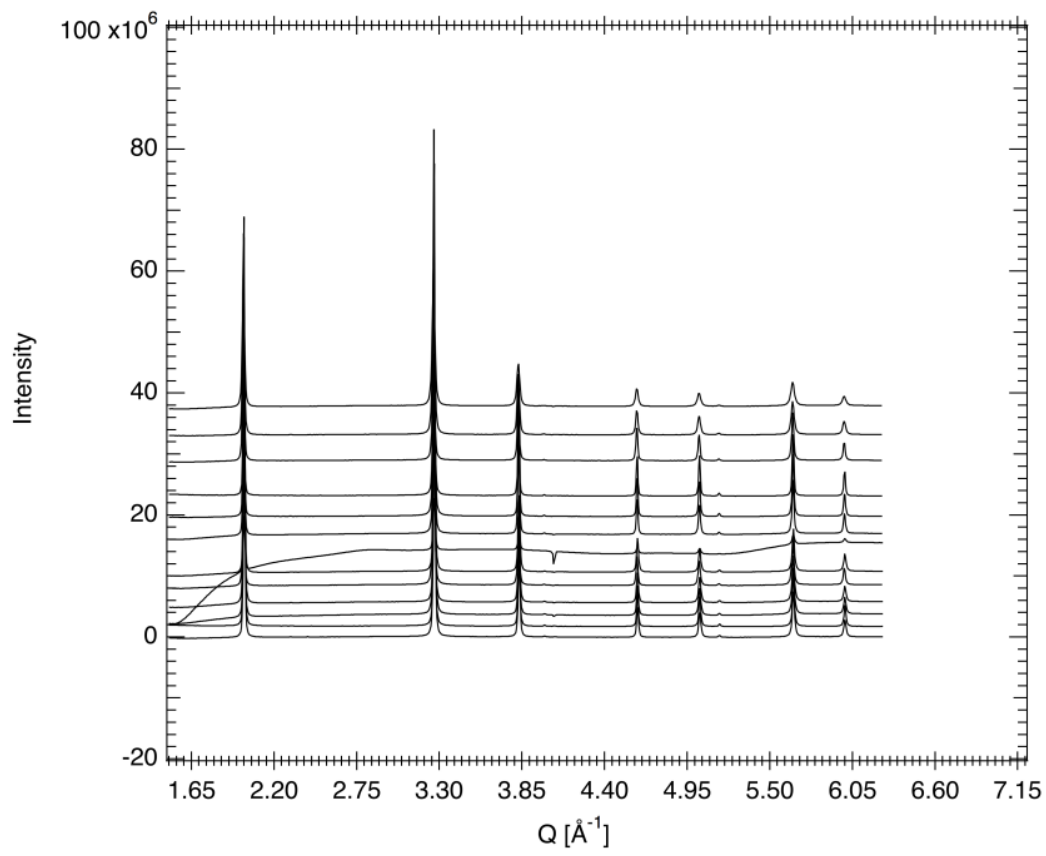

Figure S $13 \quad$ WAXS spectra for TCF22.

\section{Detailed (U)SAXS Results}

Table S 3 Overview of fitted results for (U)SAXS data for CMF4. WAXS = Wide Angle X-ray Scattering.

\begin{tabular}{|ccrrrr}
$\begin{array}{c}\text { Point } \\
\text { number }\end{array}$ & $\begin{array}{c}\text { Phase } \\
\text { identified } \\
\text { by WAXS }\end{array}$ & x & $\begin{array}{c}\text { Mean } \\
\text { diameter } \\
{[\mu \mathrm{m}]}\end{array}$ & $\begin{array}{c}\text { Median } \\
\text { Diameter } \\
{[\mu \mathrm{m}]}\end{array}$ & Porosity [\%] \\
\hline 1 & $\mathrm{FI}$ & 96.9 & 1.5 & 0.2 & 6.8 \\
2 & $\mathrm{FI}$ & 98 & 2.1 & 1.0 & 2.4 \\
3 & Mix & 99 & 1.9 & 2.0 & 1.0 \\
4 & Calc & 100.1 & 2.4 & 1.4 & 0.7 \\
5 & Calc & 101.2 & 2.2 & 1.0 & 1.1 \\
6 & Calc & 102.2 & 2.4 & 1.4 & 0.8 \\
7 & Calc & 103.3 & 2.1 & 0.9 & 0.9 \\
8 & Calc & 104.4 & 1.9 & 0.9 & 0.9 \\
9 & Calc & 105.5 & 2.9 & 2.3 & 1.0 \\
10 & Calc & 106.5 & 2.2 & 1.1 & 1.0 \\
11 & Calc & 107.6 & 2.5 & 1.5 & 1.3 \\
12 & Mix & 108.7 & 2.9 & 2.3 & 1.3 \\
13 & FI & 109.7 & 1.4 & 0.1 & 9.0 \\
14 & FI & 110.8 & 0.5 & 0.1 & 8.2 \\
\hline
\end{tabular}


Table S $4 \quad$ Overview of fitted results for (U)SAXS data for CMF8.

\begin{tabular}{|cclrrrr}
$\begin{array}{c}\text { Point } \\
\text { number }\end{array}$ & $\mathrm{x}$ & $\begin{array}{c}\text { Phase } \\
\text { identified } \\
\text { by WAXS }\end{array}$ & $\begin{array}{c}\text { Median } \\
\text { Diameter } \\
{[\mu \mathrm{m}]}\end{array}$ & $\begin{array}{c}\text { Mean } \\
\text { diameter } \\
{[\mu \mathrm{m}]}\end{array}$ & Porosity [\%] \\
\hline 1 & 96.7 & $\mathrm{FI}$ & 0.1 & 1.7 & 10.6 \\
2 & 97.8 & $\mathrm{FI}$ & 1.5 & 2.2 & 6.9 \\
3 & 98.9 & $\mathrm{FI}$ & 2.5 & 3.2 & 3.9 \\
4 & 101 & Calc & 2.4 & 3.0 & 1.5 \\
5 & 102.1 & Calc & 1.6 & 2.6 & 0.7 \\
6 & 103.2 & Calc & 1.5 & 2.5 & 0.7 \\
7 & 104.2 & Calc & 2.1 & 2.8 & 0.8 \\
8 & 105.3 & Calc & 1.7 & 2.5 & 1.3 \\
9 & 106.4 & Calc & 0.9 & 2.0 & 0.6 \\
10 & 107.4 & Calc & 2.7 & 3.2 & 2.6 \\
11 & 108.5 & Calc & 2.3 & 2.7 & 7.1 \\
12 & 109.6 & FI & 0.1 & 1.4 & 9.9 \\
13 & 110.7 & FI & 0.1 & 0.7 & 9.4 \\
\hline
\end{tabular}

Table S 5 Overview of fitted results for (U)SAXS data for CMF22.

\begin{tabular}{|c|c|c|c|c|c|}
\hline $\begin{array}{c}\text { Point } \\
\text { number }\end{array}$ & $\begin{array}{c}\text { Phase } \\
\text { identified } \\
\text { by WAXS }\end{array}$ & $x$ & $\begin{array}{c}\text { Mean } \\
\text { diameter } \\
{[\mu \mathrm{m}]}\end{array}$ & $\begin{array}{c}\text { Median } \\
\text { Diameter } \\
{[\mu \mathrm{m}]}\end{array}$ & Porosity [\%] \\
\hline 1 & $\mathrm{FI}$ & 19 & 1.7 & 0.2 & 11.7 \\
\hline 2 & $\mathrm{FI}$ & 20 & 2.6 & 1.9 & 4.2 \\
\hline 3 & $\mathrm{FI}$ & 21.1 & 3.2 & 2.7 & 2.4 \\
\hline 4 & Calc & 22.2 & 2.9 & 2.1 & 0.7 \\
\hline 5 & Calc & 23.3 & 2.8 & 2.0 & 0.8 \\
\hline 6 & Calc & 24.3 & 2.9 & 2.2 & 0.8 \\
\hline 7 & Calc & 25.4 & 2.9 & 2.1 & 0.9 \\
\hline 8 & Calc & 26.5 & 2.7 & 1.8 & 0.8 \\
\hline 9 & Calc & 27.5 & 2.3 & 1.3 & 0.9 \\
\hline 10 & Calc & 28.6 & 2.9 & 2.2 & 1.0 \\
\hline 11 & Calc & 29.7 & 2.7 & 1.8 & 1.0 \\
\hline 12 & Calc & 30.8 & 2.6 & 2.1 & 4.2 \\
\hline 13 & $\mathrm{FI}$ & 31.8 & 1.7 & 0.2 & 9.3 \\
\hline 14 & $\mathrm{FI}$ & 32.9 & 0.8 & 0.1 & 6.7 \\
\hline
\end{tabular}


Table S $6 \quad$ Overview of fitted results for (U)SAXS data for TCF8.

\begin{tabular}{|cccrrr}
$\begin{array}{c}\text { Point } \\
\text { number }\end{array}$ & $\mathrm{X}$ & $\begin{array}{c}\text { Phase } \\
\text { identified } \\
\text { by WAXS }\end{array}$ & $\begin{array}{c}\text { Median } \\
\text { Diameter } \\
{[\mu \mathrm{m}]}\end{array}$ & $\begin{array}{c}\text { Mean } \\
\text { diameter } \\
{[\mu \mathrm{m}]}\end{array}$ & Porosity [\%] \\
\hline 1 & 96.5 & $\mathrm{FI}$ & 0.5 & 1.5 & 7.1 \\
2 & 97.5 & $\mathrm{FI}$ & 1.0 & 2.1 & 8.8 \\
3 & 98.6 & $\mathrm{FI}$ & 1.6 & 2.6 & 10.5 \\
4 & 99.6 & $\mathrm{FI}$ & 2.0 & 2.9 & 9.6 \\
5 & 100.6 & $\mathrm{FI}$ & 2.2 & 3.0 & 7.8 \\
6 & 101.7 & $\mathrm{FI}$ & 2.2 & 2.8 & 9.7 \\
7 & 102.7 & $\mathrm{FI}$ & 1.4 & 2.3 & 9.4 \\
8 & 103.7 & $\mathrm{FI}$ & 1.8 & 2.6 & 8.9 \\
9 & 104.8 & $\mathrm{FI}$ & 2.0 & 2.7 & 8.0 \\
10 & 105.8 & $\mathrm{FI}$ & 2.3 & 3.1 & 9.6 \\
11 & 106.8 & $\mathrm{FI}$ & 1.9 & 2.7 & 8.7 \\
12 & 107.9 & $\mathrm{FI}$ & 1.7 & 2.6 & 9.9 \\
13 & 108.9 & $\mathrm{FI}$ & 1.2 & 2.2 & 10.1 \\
14 & 110 & $\mathrm{FI}$ & 0.3 & 1.3 & 8.1 \\
\hline
\end{tabular}

Table S $7 \quad$ Overview of fitted results for (U)SAXS data for TCF4.

\begin{tabular}{|cccrrr}
$\begin{array}{c}\text { Point } \\
\text { number }\end{array}$ & $\mathrm{X}$ & $\begin{array}{c}\text { Phase } \\
\text { identified } \\
\text { by WAXS }\end{array}$ & $\begin{array}{c}\text { Median } \\
\text { Diameter } \\
{[\mu \mathrm{m}]}\end{array}$ & $\begin{array}{c}\text { Mean } \\
\text { diameter } \\
{[\mu \mathrm{m}]}\end{array}$ & Porosity [\%] \\
\hline 1 & 99.8 & $\mathrm{FI}$ & 1.6 & 2.6 & 10.0 \\
2 & 100.8 & $\mathrm{FI}$ & 1.9 & 2.7 & 9.4 \\
3 & 101.9 & $\mathrm{FI}$ & 1.9 & 2.6 & 10.5 \\
4 & 102.9 & $\mathrm{FI}$ & 1.6 & 2.4 & 9.4 \\
5 & 103.9 & $\mathrm{FI}$ & 0.9 & 2.0 & 9.0 \\
6 & 105 & $\mathrm{FI}$ & 1.2 & 2.1 & 9.5 \\
7 & 106 & $\mathrm{FI}$ & 1.4 & 2.4 & 10.0 \\
8 & 107.1 & $\mathrm{FI}$ & 1.4 & 2.4 & 9.4 \\
9 & 108.1 & $\mathrm{FI}$ & 1.3 & 2.3 & 9.2 \\
10 & 109.1 & $\mathrm{FI}$ & 1.4 & 2.3 & 10.5 \\
11 & 110.2 & $\mathrm{FI}$ & 0.5 & 1.5 & 8.6 \\
12 & 111.2 & $\mathrm{FI}$ & 0.1 & 1.1 & 8.6 \\
\hline
\end{tabular}


Table S $8 \quad$ Overview of fitted results for (U)SAXS data for TCF22.

\begin{tabular}{|cccrrr}
$\begin{array}{c}\text { Point } \\
\text { number }\end{array}$ & $\mathrm{X}$ & $\begin{array}{c}\text { Phase } \\
\text { identified } \\
\text { by WAXS }\end{array}$ & $\begin{array}{c}\text { Median } \\
\text { Diameter } \\
{[\mu \mathrm{m}]}\end{array}$ & $\begin{array}{c}\text { Mean } \\
\text { diameter } \\
{[\mu \mathrm{m}]}\end{array}$ & Porosity [\%] \\
\hline 1 & 20 & $\mathrm{FI}$ & 0.5 & 1.6 & 9.9 \\
2 & 21.1 & $\mathrm{FI}$ & 1.2 & 2.3 & 9.9 \\
3 & 22.1 & $\mathrm{FI}$ & 1.5 & 2.3 & 10.5 \\
4 & 23.1 & $\mathrm{FI}$ & 1.0 & 1.1 & 9.5 \\
5 & 24.2 & $\mathrm{FI}$ & 1.3 & 2.2 & 9.8 \\
6 & 25.7 & $\mathrm{FI}$ & 1.2 & 2.1 & 9.7 \\
7 & 26.3 & $\mathrm{FI}$ & 1.1 & 2.1 & 9.7 \\
8 & 27.3 & $\mathrm{FI}$ & 1.0 & 2.1 & 10.5 \\
9 & 28.3 & $\mathrm{FI}$ & 1.3 & 2.2 & 9.4 \\
10 & 29.4 & $\mathrm{FI}$ & 1.6 & 2.4 & 8.5 \\
11 & 30.4 & $\mathrm{FI}$ & 1.6 & 2.4 & 7.7 \\
12 & 32.5 & $\mathrm{FI}$ & 0.3 & 1.3 & 6.6 \\
13 & 33.5 & $\mathrm{FI}$ & 0.1 & 1.1 & 5.9 \\
\hline
\end{tabular}

Table S $9 \quad$ Overview of fitted (U)SAXS data for WDF4.

\begin{tabular}{|ccrrrr}
\hline $\begin{array}{c}\text { Point } \\
\text { number }\end{array}$ & $\begin{array}{c}\text { Phase } \\
\text { identified } \\
\text { by WAXS }\end{array}$ & x & $\begin{array}{c}\text { Mean } \\
\text { diameter } \\
{[\mu \mathrm{m}]}\end{array}$ & $\begin{array}{c}\text { Median } \\
\text { Diameter } \\
{[\mu \mathrm{m}]}\end{array}$ & Porosity [\%] \\
\hline 1 & Calc & 96.5 & 3.0 & 2.6 & 0.8 \\
2 & Calc & 97.5 & 2.9 & 2.5 & 0.8 \\
3 & Calc & 98.5 & 3.1 & 2.6 & 0.8 \\
4 & Calc & 99.5 & 3.3 & 3.0 & 0.9 \\
5 & Calc & 100.5 & 2.9 & 2.3 & 0.9 \\
6 & Calc & 101.6 & 2.6 & 1.8 & 0.8 \\
7 & Calc & 102.6 & 1.8 & 0.3 & 0.8 \\
8 & Calc & 103.6 & 3.0 & 2.7 & 1.0 \\
9 & Calc & 104.6 & 3.1 & 2.8 & 1.0 \\
10 & Calc & 105.6 & 2.9 & 2.5 & 0.8 \\
11 & Calc & 106.8 & 2.6 & 1.8 & 0.9 \\
12 & Calc & 107.7 & 2.8 & 2.4 & 1.1 \\
13 & Calc & 108.7 & 3.0 & 3.0 & 1.3 \\
14 & Calc & 109.7 & 2.7 & 2.7 & 2.0 \\
\hline
\end{tabular}


Table S $10 \quad$ Overview of fitted results for (U)SAXS data for WDF8.

\begin{tabular}{|ccrcrr|}
\hline $\begin{array}{c}\text { Point } \\
\text { number }\end{array}$ & $\begin{array}{c}\text { Phase } \\
\text { identified } \\
\text { by WAXS }\end{array}$ & x & $\begin{array}{c}\text { Mean diameter } \\
{[\mu \mathrm{m}]}\end{array}$ & $\begin{array}{c}\text { Median Diameter } \\
{[\mu \mathrm{m}]}\end{array}$ & Porosity [\%] \\
\hline 1 & Calc & 97.7 & 3.3 & 3.0 & 1.1 \\
2 & Calc & 98.8 & 3.1 & 2.7 & 0.8 \\
3 & Calc & 99.9 & 3.2 & 2.9 & 0.9 \\
4 & Calc & 100.8 & 3.2 & 2.8 & 1.0 \\
5 & Calc & 101.9 & 3.1 & 2.8 & 1.0 \\
6 & Calc & 102.9 & 3.1 & 2.7 & 0.9 \\
7 & Calc & 103.9 & 3.3 & 3.4 & 1.0 \\
8 & Calc & 105 & 3.1 & 2.6 & 0.8 \\
9 & Calc & 106 & 2.7 & 2.1 & 0.8 \\
10 & Calc & 107 & 3.2 & 3.1 & 0.8 \\
11 & Calc & 108.1 & 3.0 & 2.5 & 0.9 \\
12 & Calc & 109.1 & 2.9 & 2.5 & 1.8 \\
13 & Calc & 110.2 & 2.0 & 0.7 & 0.9 \\
\hline
\end{tabular}

Table S 11 Overview of fitted results for (U)SAXS data for WDF22.

\begin{tabular}{|c|c|c|c|c|c|}
\hline $\begin{array}{l}\text { Point } \\
\text { number }\end{array}$ & $\begin{array}{c}\text { Phase } \\
\text { identified by } \\
\text { WAXS }\end{array}$ & $x$ & $\begin{array}{c}\text { Mean } \\
\text { diameter } \\
{[\mu \mathrm{m}]}\end{array}$ & $\begin{array}{c}\text { Median } \\
\text { Diameter } \\
{[\mu \mathrm{m}]}\end{array}$ & Porosity [\%] \\
\hline 1 & Calc & 18.5 & 3.2 & 2.8 & 1.0 \\
\hline 2 & Calc & 19.5 & 3.2 & 2.8 & 0.9 \\
\hline 3 & Calc & 20.6 & 3.4 & 3.1 & 1.0 \\
\hline 4 & Calc & 21.7 & 2.8 & 2.2 & 0.8 \\
\hline 5 & Calc & 22.8 & 2.7 & 2.2 & 0.9 \\
\hline 6 & Calc & 23.8 & 2.7 & 2.2 & 0.9 \\
\hline 7 & Calc & 24.8 & 2.9 & 2.7 & 1.1 \\
\hline 8 & Calc & 26 & 2.6 & 2.1 & 1.2 \\
\hline 9 & Calc & 27 & 2.7 & 2.2 & 1.2 \\
\hline 10 & Calc & 28.1 & 2.9 & 2.3 & 0.9 \\
\hline 11 & Calc & 29.2 & 3.2 & 2.9 & 1.0 \\
\hline 12 & Calc & 30.3 & 3.0 & 2.5 & 0.9 \\
\hline 13 & Calc & 31.3 & 2.4 & 1.6 & 1.2 \\
\hline
\end{tabular}




\section{Detailed (U)SANS Results}

Table S 12 Overview of detailed (U)SANS Fitting results.

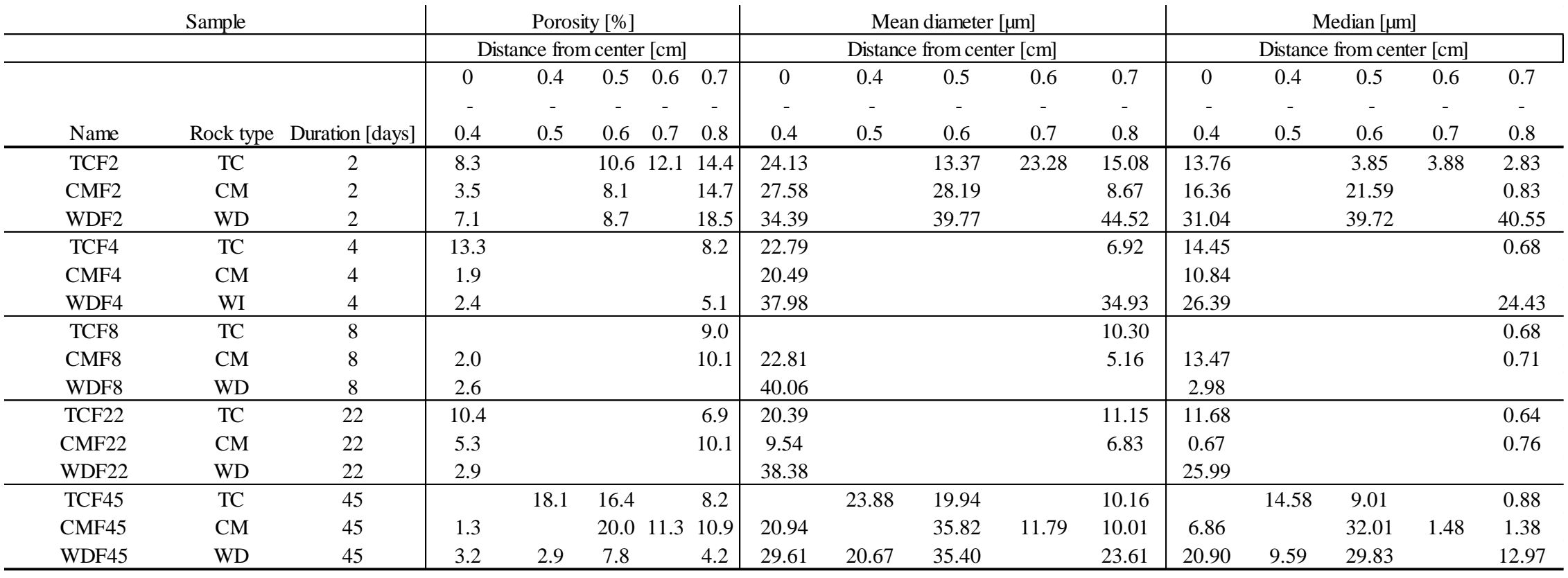




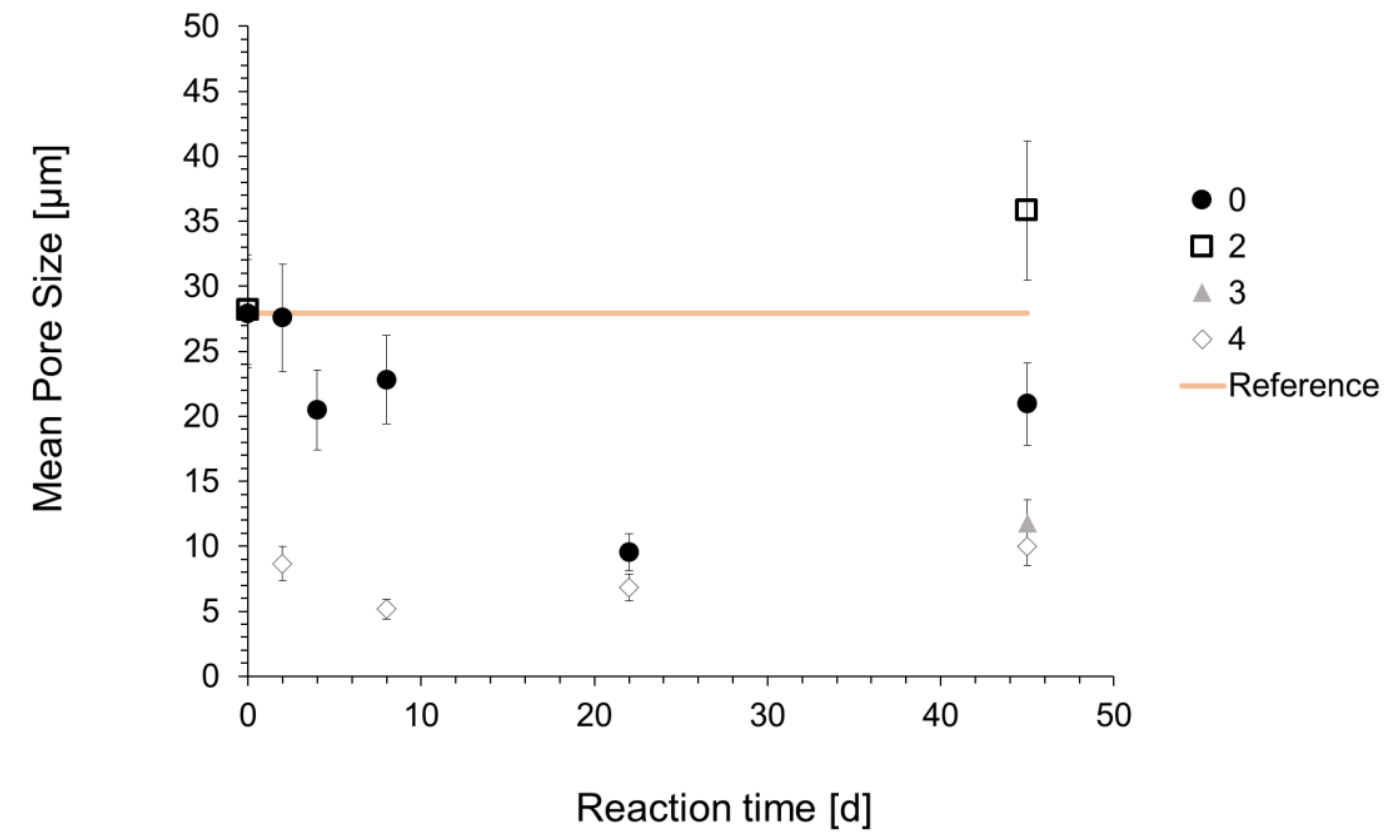

Figure S 14 Mean Porosity Evolution for low porosity CM limestone. 
TC Mean Pore Size Changes

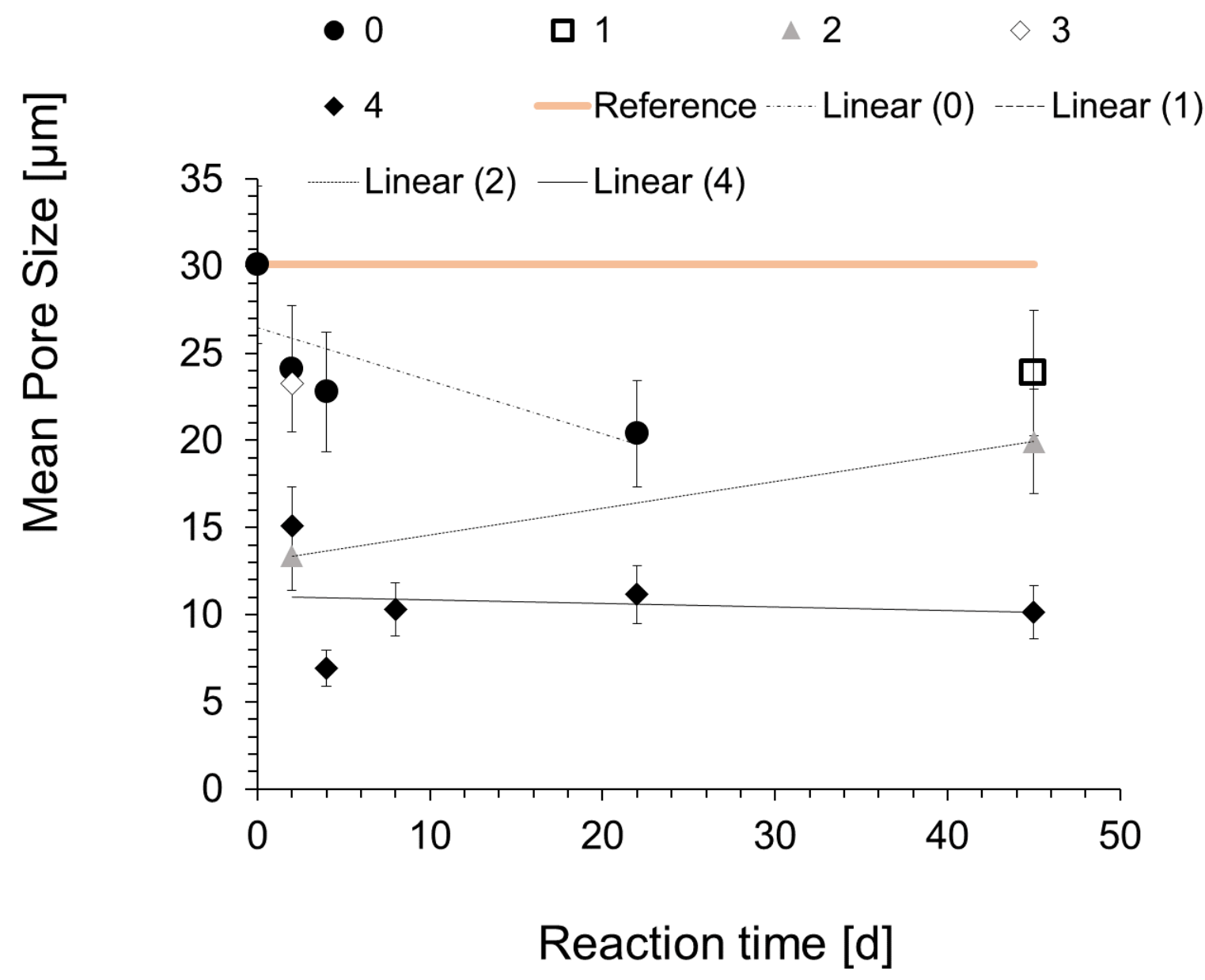

Figure S 15 Evolution of the Mean Pore Size for high porosity TC limestone. 


\section{Pore Size Distribution for TC Limestone}
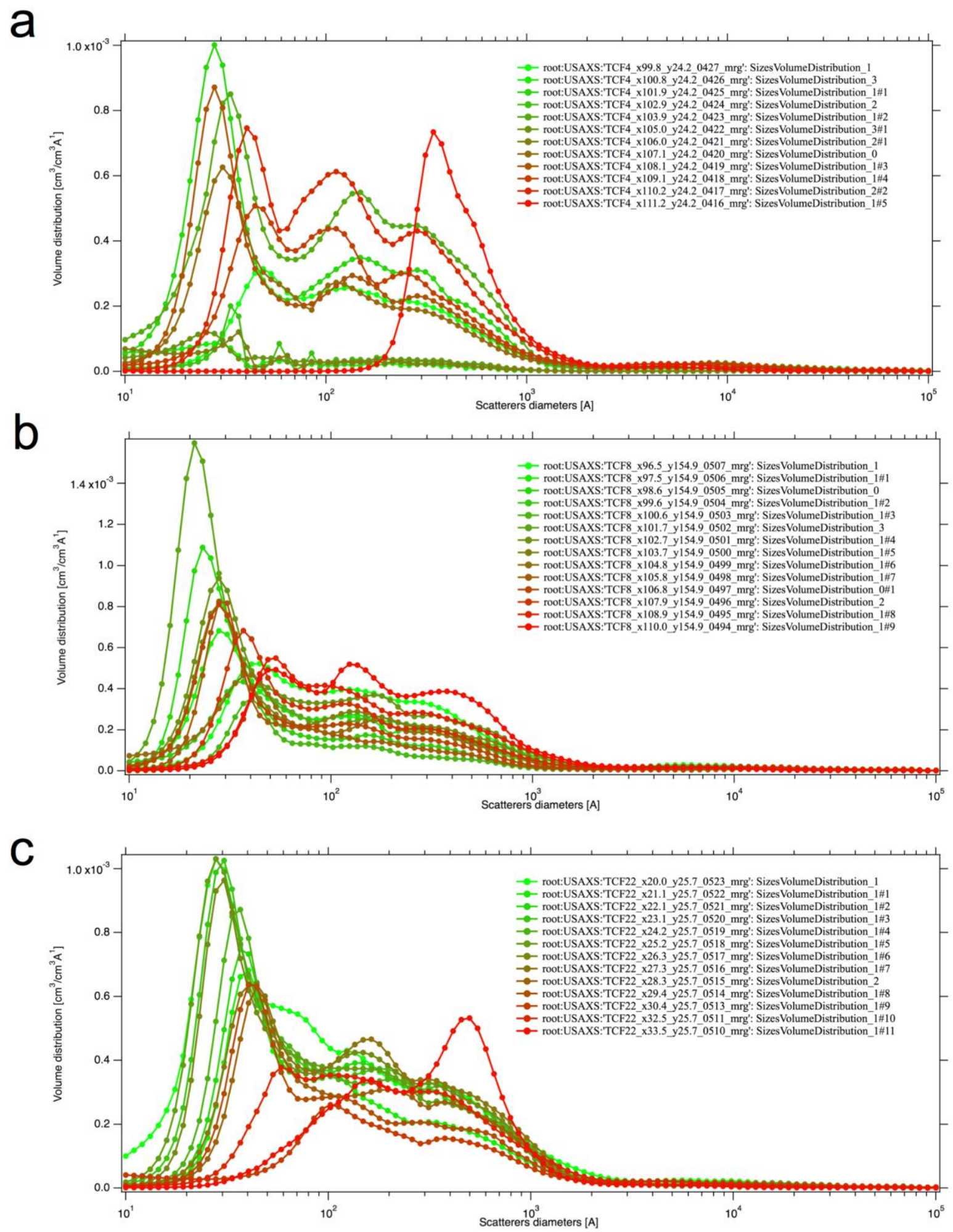

Figure $\mathrm{S} 16$

Pore Size Distribution for (U)SAXS measurements on TC limestone reacted for (a) 4 days (b) 8 days and (c) 22 days 


\section{Additional SEM Characterization}
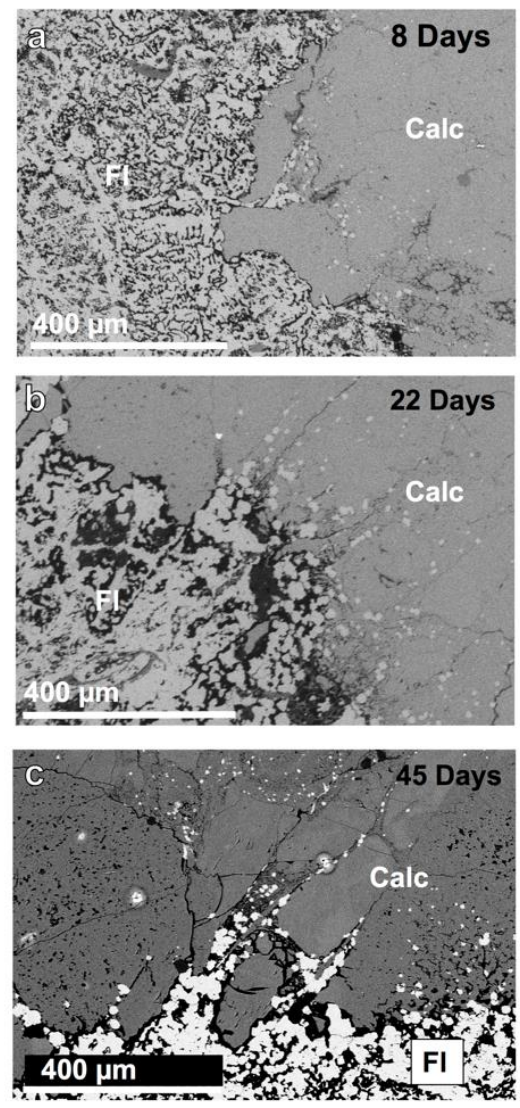

Figure S 17 BSE SEM images of the CM limestone reacted for (a) 8 days, (b) 22 days and (c) 45 days. Enhanced replacement along the grain boundaries is visible. 

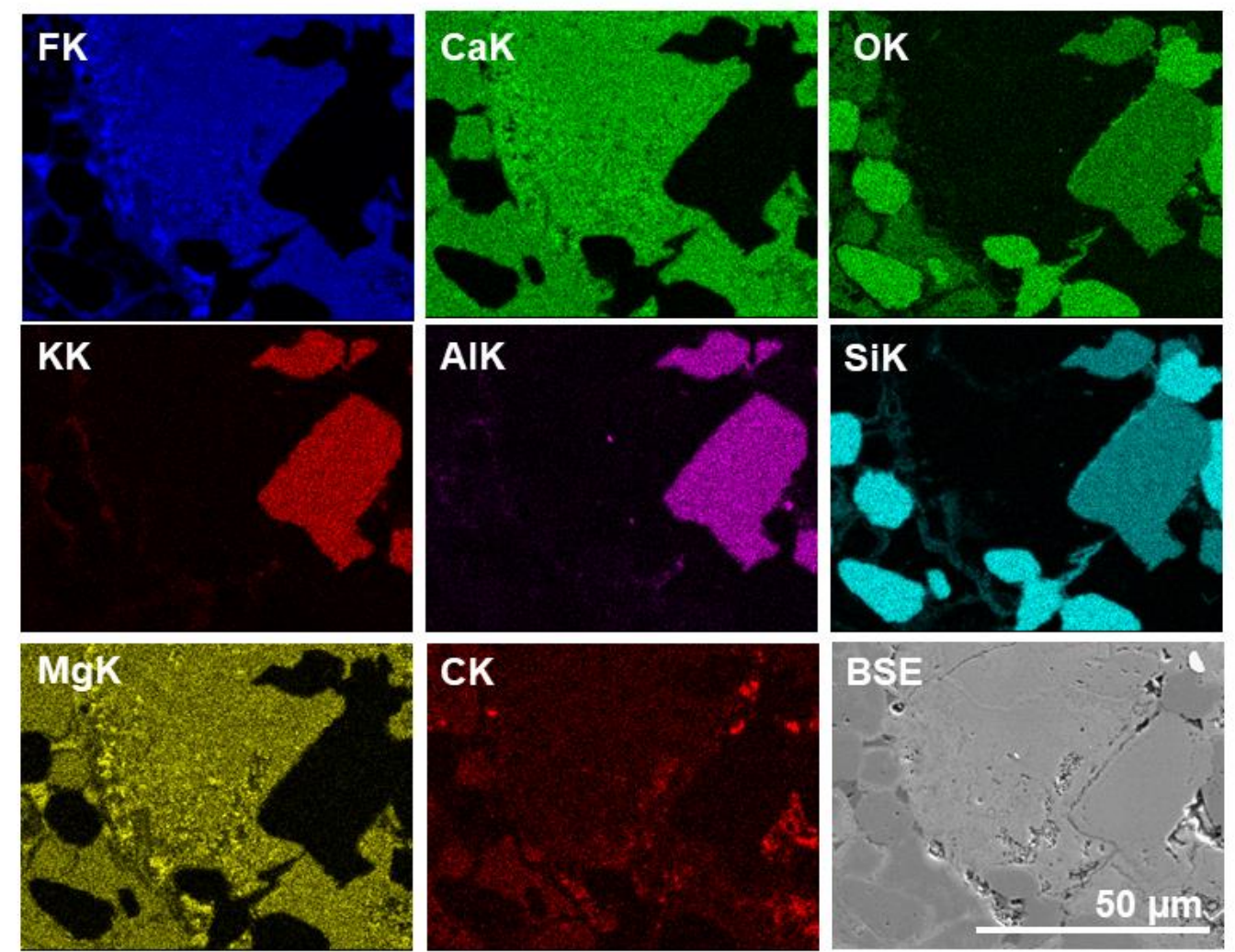

Figure S 18

SEM-EDS mapping of WDF45.
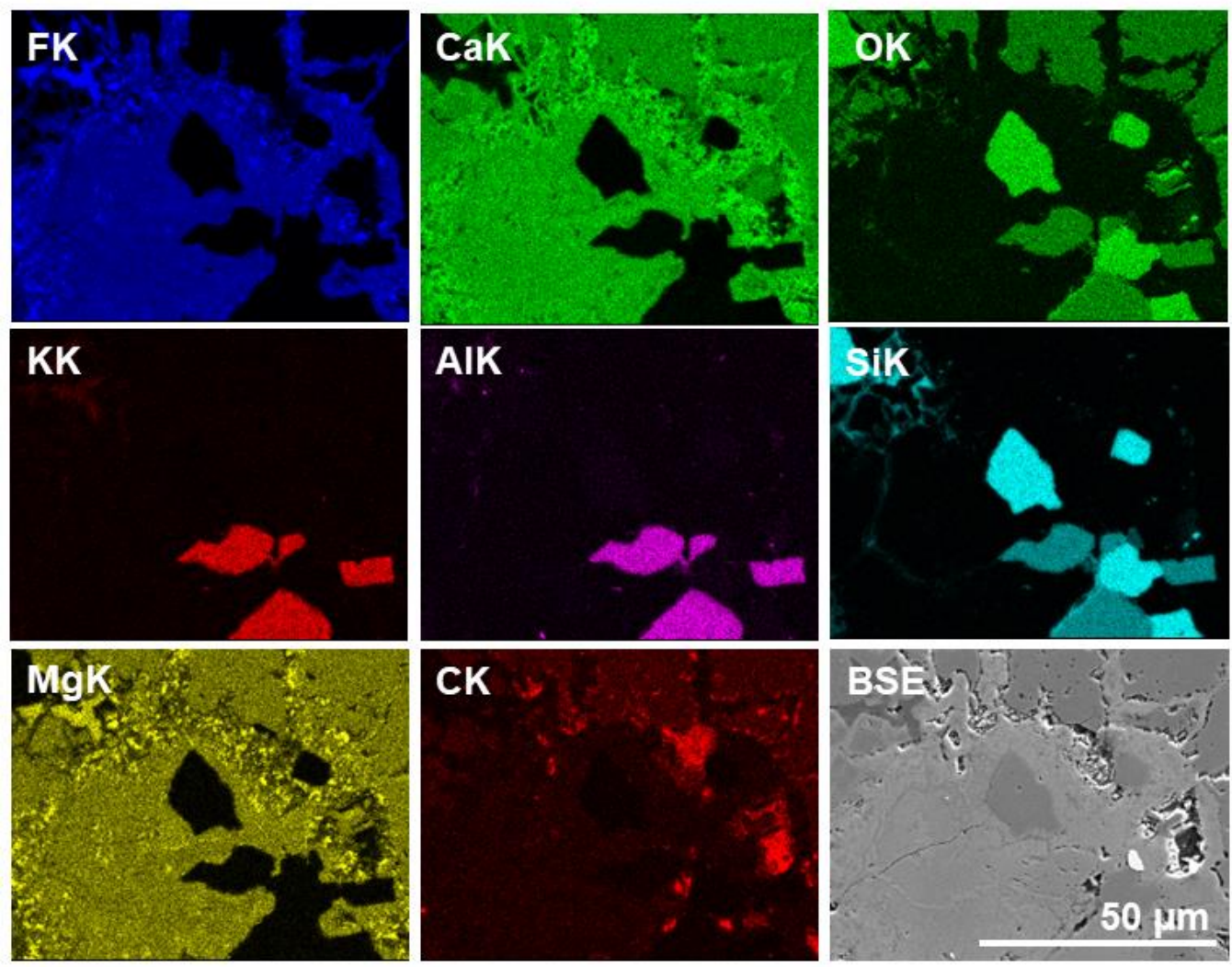

Figure S $19 \quad$ SEM-EDS mapping of WDF45. 


\section{Additional ToF-SIMS characterization}
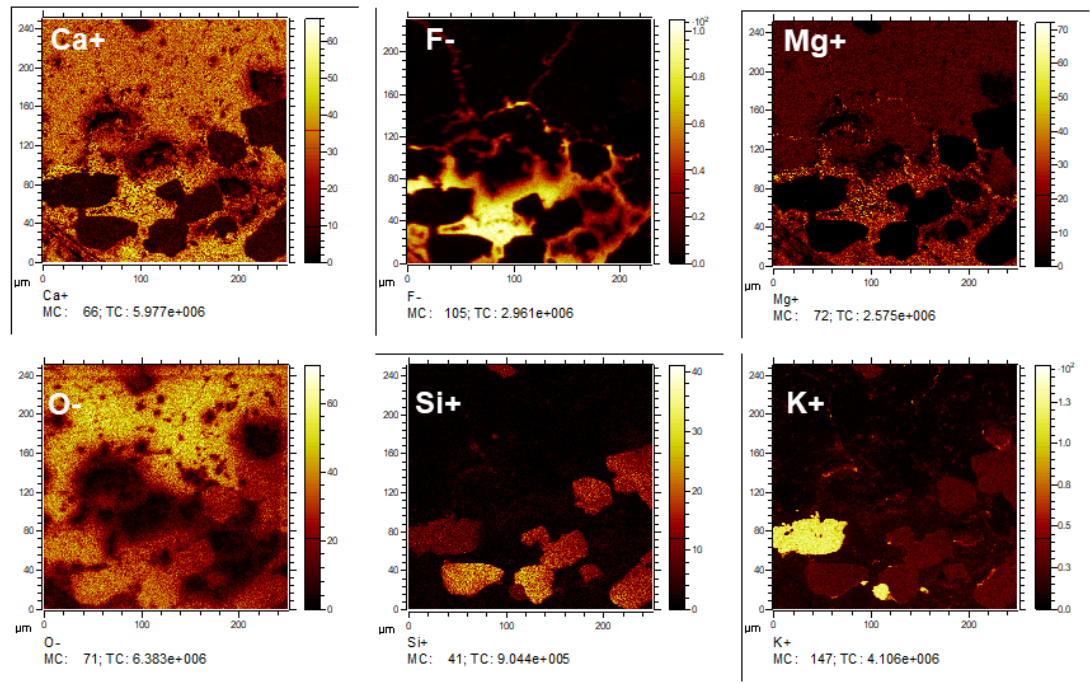

Figure S $20 \quad$ ToF-SIMS elemental maps of WDF45 sample. 
13. Images Used in Rim Thickness Analyses
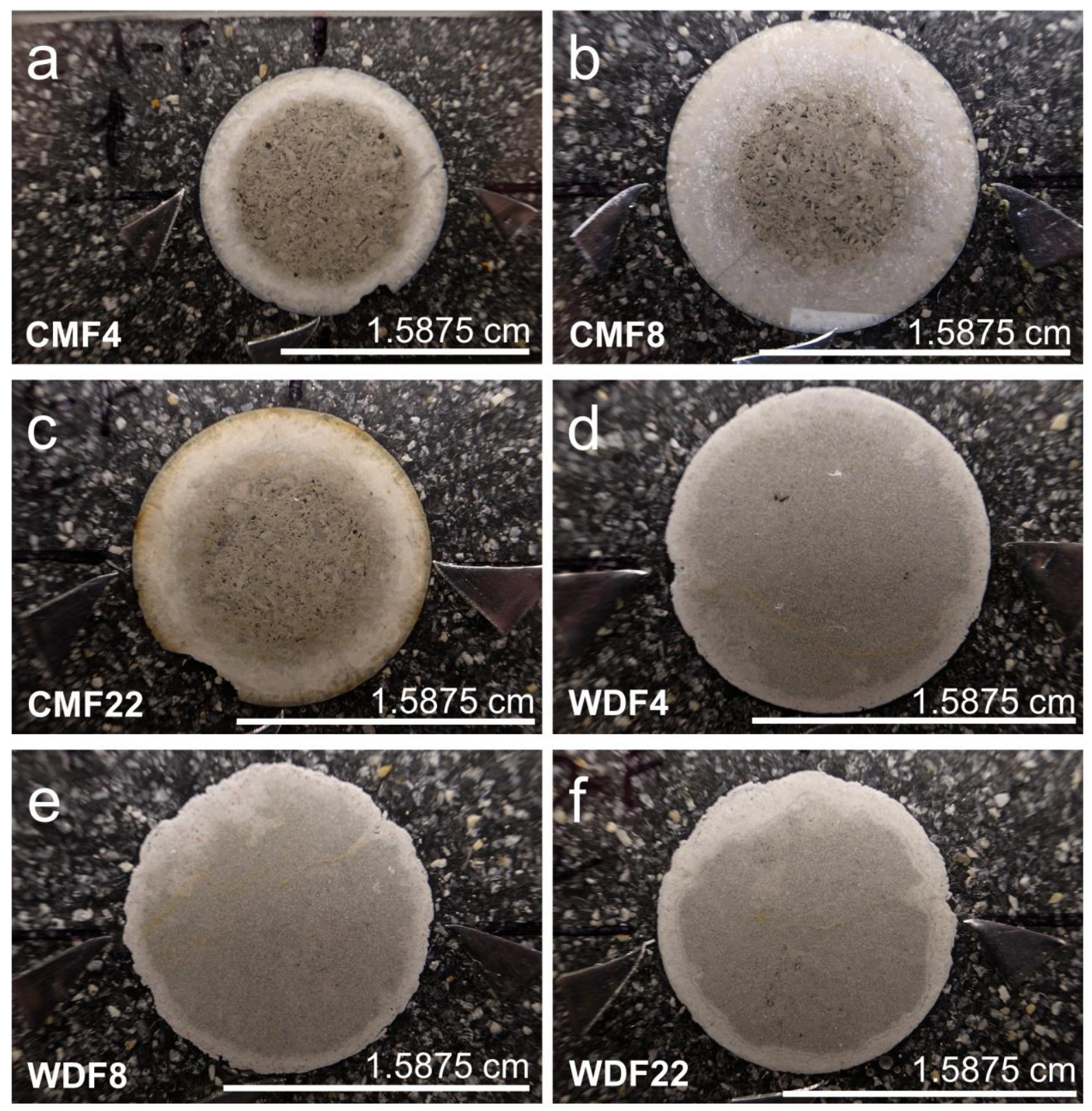

Figure S21

Images used for rim thickness image analyses. (a) CMF (b) CMF8 (c) CMF22 (d) WDF4 (e) WDF8 (f) WDF22. 